\title{
Developing a safe and effective CAR T-cell immunotherapy for breast cancer: progress and pitfalls
}

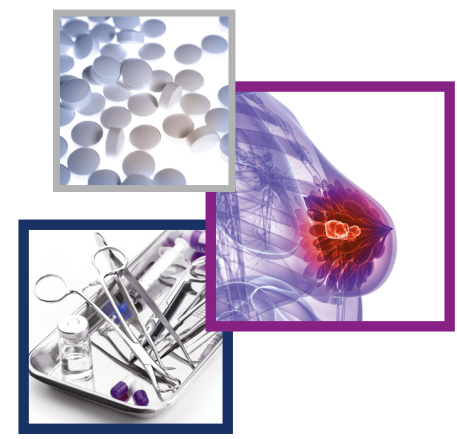

\author{
Pierre Antoine*,1 (iD) \& John Maher ${ }^{1,2,3,4}$ \\ ${ }^{1}$ King's College London, School of Cancer \& Pharmaceutical Sciences, Guy's Cancer Centre, Great Maze Pond, London, SE1 9RT, \\ UK \\ 2Leucid Bio Ltd., Guy's Hospital, Great Maze Pond, London, SE1 9RT, UK \\ ${ }^{3}$ Department of Clinical Immunology \& Allergy, King's College Hospital NHS Foundation Trust, Denmark Hill, London, SE5 9RS, UK \\ ${ }^{4}$ Department of Immunology, Eastbourne Hospital, Kings Drive, Eastbourne, East Sussex, BN21 2UD, UK \\ *Author for correspondence: pierre.antoine@kcl.ac.uk
}

\section{Practice points}

- Several antigens have been identified as potential targets for chimeric antigen receptors (CAR) T-cell therapy in breast cancer preclinical models (Table 1).

- CAR T cells are currently being investigated in Phase I/II trials in patients with breast cancer (Table 2).

- Strategies to improve CAR T-cell specificity for malignant cells are currently being studied to increase safety.

- For CAR T cells to retain functionality in the immunosuppressive tumor microenvironment, innovative designs disrupting the recognition of inhibitory signals are being investigated.

- Strategies to increase persistence of CAR T cells and trafficking to the tumor are currently being explored to achieve prolonged antitumor protection.

- More relevant preclinical models based on a better understanding of the biology of breast cancer and CAR T cells will be key to the future development of CAR T-cell therapy for solid tumors.

Current targeted therapies for breast cancer include hormone inhibitors, monoclonal antibodies and tyrosine kinase inhibitors. However, a significant unmet therapeutic need remains for refractory disease and in particular for the triple negative subtype, which lacks hormone receptors and HER2. Chimeric antigen receptors $\mathrm{T}$ cells are genetically engineered to deploy selective cytolytic activity against cells that express cognate native target. Durable remissions have been achieved in refractory hematological malignancies but similar success against solid tumors remains elusive. Several hurdles hinder progress, including the need to identify safe antigens, promote T-cell homing to tumor sites and to ensure the persistence of functional chimeric antigen receptors $\mathrm{T}$ cells within the immunosuppressive tumor microenvironment. Perspectives to enable the attainment of this goal are presented in this review.

First draft submitted: 24 February 2020; Accepted for publication: 23 July 2020; Published online: 18 September 2020

Keywords: adoptive T-cell therapy • breast cancer $\bullet$ CAR T cells • ErbB1 • HER2 • MUC1 • TNBC

Breast cancer is the most frequent malignancy in women. Besides standard treatment approaches (surgery, chemotherapy, radiotherapy and hormonal blockade), new targeted therapies have been developed that exploit our improved understanding of the underlying biology of these tumors. Nonetheless, not all patients benefit from these interventions and some subtypes such as triple negative disease remain particularly difficult to treat. Chimeric antigen receptors (CAR) are fusion proteins that couple the binding of a native tumor-associated target to the delivery of a bespoke T-cell activating signal. Targeting is most commonly achieved using a single chain antibody

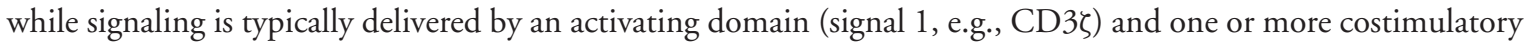
modules (signal 2). CAR T cells have induced durable clinical responses in treatment refractory hematological malignancies, but similar results have not been achieved thus far in solid tumors. Limited intratumoral trafficking and a panoply of immunosuppressive signals delivered within the tumor microenvironment (TME) play key roles 
in impairing CAR T cell function in solid tumors. Strategies aiming at circumventing these hurdles are a major focus of current CAR T-cell development.

\section{Candidate CAR T cell targets in breast cancer}

Targets that are currently being studied for CAR T-cell treatment of breast cancer include tumor-associated antigens (TAA), neoantigens, NKG2D ligands and stromal cell markers. Ideal TAA are surface molecules that are overexpressed on transformed cells in a large proportion of patients. TAA should also be absent or minimally expressed on nonmalignant cells in order to minimize the risk of on-target off-tumor toxicity. Finally, the toxicity following immune recognition of normal cells by CAR T cells should be manageable. As an example, hypogammaglobulinemia which commonly results from anti-CD19 treatment in B cell malignancies is readily controlled by regular infusion of pooled immunoglobulin. Neoantigens are alternative targets originating from oncogenic viral proteins, somatic mutations or gene rearrangements in malignant cells. Neoantigens are only expressed by malignant cells which makes them a safer target for CAR T-cell therapy. Several TAA and neoantigens have been identified in breast cancer and, among these, molecules driving tumorigenesis, tumor invasion and metastasis represent particularly attractive targets. In the paragraphs that follow, a number of potential targets will be discussed. Safety aspects associated with these targets is considered in the section that follows thereafter. All the studies cited in this review and investigating CAR T cells targeting relevant antigens for breast cancer are summarized in Table 1.

\section{ErbB family}

The ErbB family comprises the transmembrane receptors EGFR or ErbB1, ErbB2 (HER2/neu), ErbB3 and ErbB4. They are weakly but widely expressed on nonmalignant cells. However, dysregulation of the ErbB network is prevalent in a variety of breast cancer subtypes [42,43]. HER2 is overexpressed in $15-20 \%$ of tumors and is associated with poor prognosis [44]. Early studies demonstrated that first generation HER2-specific CAR T cells in which an activating domain alone was incorporated promoted target cell lysis accompanied by IL-2 production [1]. The addition of costimulatory domains to create second and third generation HER2-specific CARs led to improved tumor control in preclinical models [2]. HER2-CAR T-cell therapy is currently being tested in several Phase I/II trials (Table 2). ErbB3, the natural ligand of heregulin-1 $\beta$, heterodimerizes with HER2 and participates in signaling and poor prognosis. Heregulin-1 $\beta$-based second generation (4-1BB) CAR T cells inhibit growth of the breast cancer cell line SKBR3 cells both in vitro and in vivo in mice [21].

Triple-negative breast cancers (TNBC) lack expression of estrogen receptors, progesterone receptors and HER2 and represent $15-20 \%$ of all breast cancers. These tumors are highly aggressive and have a poor prognosis [45]. EGFR is overexpressed in $45-70 \%$ of TNBC. Third generation EGFR-specific CAR T cells have been shown to lyse TNBC breast cancer cell lines in vitro and delay the growth of both cell line derived tumors and patient derived xenografts in mice by approximately 50\% [22]. A promiscuous CAR targeting ErbB1 homo and heterodimers and the ErbB2/3 heterodimer has also displayed potent antitumor efficacy in vitro and against a range of xenografts in vivo, including a breast cancer model [23]. Due to EGFR expression on epithelial cells, systemic administration of EGFR- or panErbB targeting CAR T cells is likely to trigger severe on-target off-tumor toxicity and their use is currently limited to intra-tumoral or intra-cavity injection. The EGFR splice variant EGFRvIII, a constitutively active extracellular deletion mutant, is a neoantigen that has been mostly studied in glioblastoma. Data suggest that it could be a promising target in breast cancer as well [46]. In particular, EGFRvIII-specific CAR T cells could be a promising therapeutic option for the tamoxifen-resistant ER+ PR-subset where EGFRvIII could contribute to the estrogen-independent growth [47].

\section{MUC1}

MUC1 is another potential target that is frequently over-expressed in the majority of breast cancers, including TNBC. Cancer-associated MUC1 displays an altered glycosylation profile and loss of polarized expression making it a promising candidate for CAR T-cell therapy. To exploit this, Wilkie et al. developed a HMFG2-based CAR which delayed tumor growth in SCID Beige mice [16]. More recently, a CAR-targeted against MUC1-Tn (a specific tumorassociated glycoform) has been developed and is currently being evaluated in patients (NCT04025216; Table 2) [17]. A CAR directed against a cleaved derivative of the MUC1 extracellular domain, known as MUC1* and specifically found on the surface of tumor cells, has also been engineered and Phase I evaluation in man has recently commenced (NCT04020575; Table 2). 
Table 1. Preclinical development of chimeric antigen receptor T cells in breast cancer.

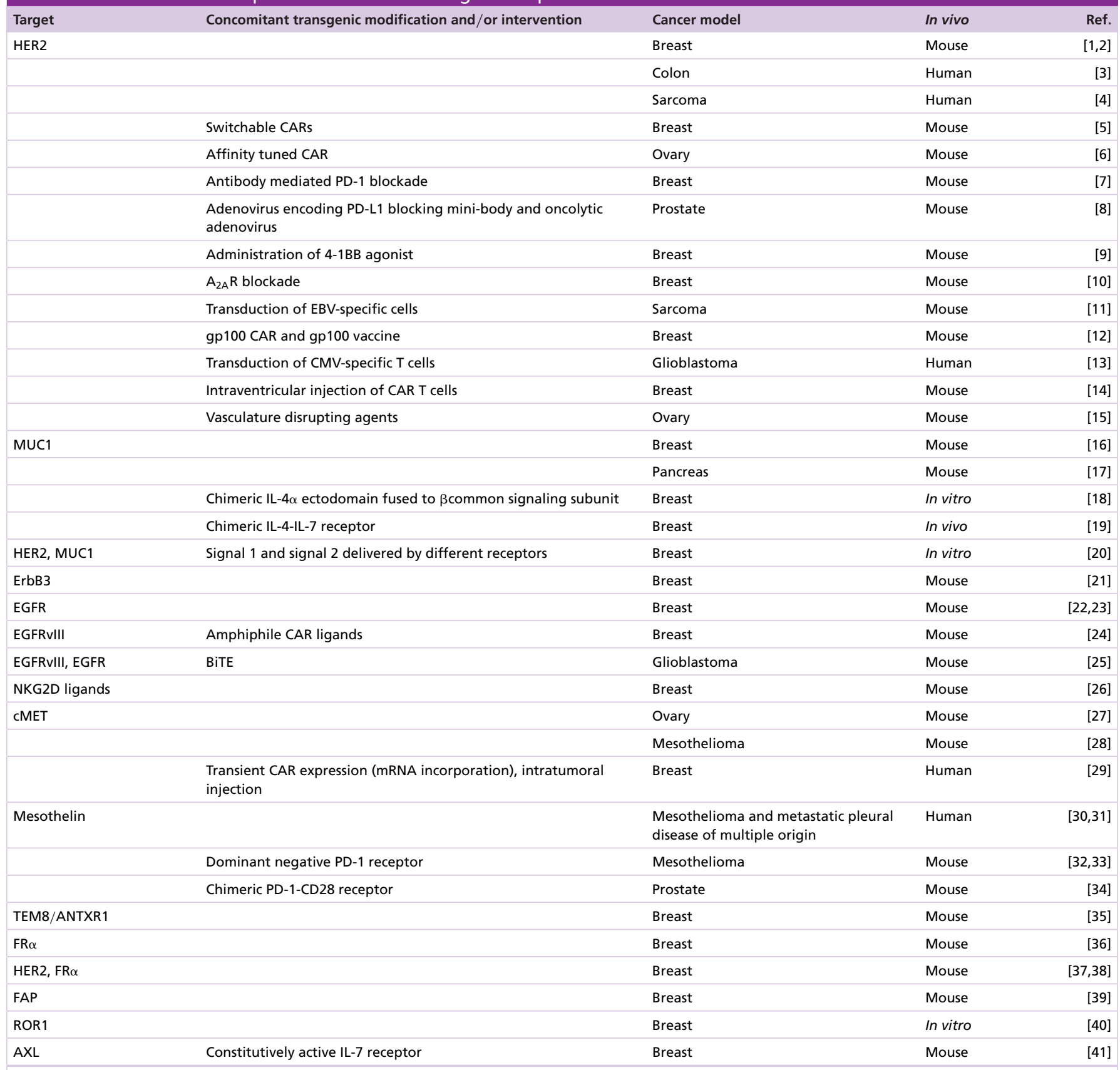

The table provides a synthetic view of studies cited in this review.

\section{NKG2D ligands}

NKG2D ligands are stress-inducible surface proteins expressed by various tumor types including TNBC. NKG2D is expressed on all NK and $\mathrm{CD}^{+} \mathrm{T}$ cells, most $\gamma \delta \mathrm{T}$ and invariant NKT cells and on a subset of CD4 ${ }^{+} \mathrm{T}$ cells [48]. NKG2D engagement activates NK cells and provides costimulation to $\mathrm{CD}^{+} \mathrm{T}$ cells through the adaptor molecule DAP10. T cells expressing a chimeric receptor comprising the extracellular domain of NKG2D and signaling through $\mathrm{CD} 3 \zeta$ and $\mathrm{CD} 27 / 4-1 \mathrm{BB}$ displayed antitumor efficacy both in vitro and in vivo in mice bearing MDA-MB-231 TNBC xenografts [26]. Celyad is developing an NKG2D-targeted CAR designated CYAD-101, which is being evaluated against colorectal cancer in trials utilizing both autologous and allogeneic $\mathrm{T}$ cells as hosts (NCT03310008, NCT03692429). A Phase I study has just started in breast cancer (NCT04107142; Table 2). 
Table 2. Chimeric antigen receptor T cell trials in breast cancer.

\begin{tabular}{|c|c|c|c|c|c|}
\hline Targeted antigen & Phases & $\begin{array}{l}\text { Enrollment (as of } 30 \\
\text { June 2020) }\end{array}$ & Institutions & Country & Identifier \\
\hline HER-2 & 1 & Recruiting & City of Hope Medical Center & USA & NCT03696030 \\
\hline HER-2 & $\frac{1}{2}$ & Completed & Fuda Cancer Hospital & China & NCT02547961 \\
\hline $\begin{array}{l}\text { HER-2, GD2, CD44v6 } \\
\text { (simultaneously) }\end{array}$ & $\frac{1}{2}$ & Recruiting & The Seventh Affiliated Hospital & China & NCT04430595 \\
\hline TnMUC1 & 1 & Recruiting & $\begin{array}{l}\text { The Angeles Clinic and Research Institute, Hospital } \\
\text { of the University of Pennsylvania }\end{array}$ & USA & NCT04025216 \\
\hline MUC1* & 1 & Recruiting & Fred Hutchinson Cancer Research Center & USA & NCT04020575 \\
\hline cMET & 1 & Completed & $\begin{array}{l}\text { Abramson Cancer Center of the University of } \\
\text { Pennsylvania }\end{array}$ & USA & NCT01837602 \\
\hline CMET & 1 & Terminated & University of Pennsylvania & USA & NCT03060356 \\
\hline Mesothelin & $\frac{1}{2}$ & Recruiting & Memorial Sloan Kettering Cancer Center & USA & NCT02414269 \\
\hline Mesothelin & 1 & Recruiting & Memorial Sloan Kettering Cancer Center & USA & NCT02792114 \\
\hline EpCAM & 1 & Recruiting & West China Hospital & China & NCT02915445 \\
\hline CD70 & $\frac{1}{2}$ & Recruiting & National Institutes of Health Clinical Center & USA & NCT02830724 \\
\hline ROR1 & 1 & $\begin{array}{l}\text { Suspended due to } \\
\text { COVID-19 }\end{array}$ & $\begin{array}{l}\text { Fred Hutch/University of Washington Cancer } \\
\text { Consortium }\end{array}$ & USA & NCT02706392 \\
\hline NKG2DL & 1 & Not yet recruiting & Landmark Medical Center & Malaysia & NCT04107142 \\
\hline CD44v6 & $\frac{1}{2}$ & Recruiting & Shenzhen Geno-Immune Medical Institute & China & NCT04427449 \\
\hline $\begin{array}{l}\text { HER-2 in combination with the } \\
\text { binary oncolytic adenovirus } \\
\text { CAdVEC }\end{array}$ & 1 & Not yet recruiting & Baylor College of Medicine & USA & NCT03740256 \\
\hline
\end{tabular}

CMET

The cMET receptor tyrosine kinase (or hepatocyte growth factor receptor) is expressed in breast cancer, including basal-like TNBC [49]. Frigault et al. developed a second generation anti-cMET CAR that lyses cMET ${ }^{+}$SKOV3 cells both in vitro and in vivo [27]. An alternative cMET-specific CAR was described by Thayaparan and demonstrated antitumor activity in models of malignant mesothelioma [28].

\section{Mesothelin}

Mesothelin (MSLN) was first identified in ovarian cancer but is also expressed in a significant proportion of TNBC [50]. Expression by normal cells is limited to mesothelium [51]. Aberrant MSLN expression plays a role in malignant transformation and cancer aggressiveness as it promotes proliferation, local invasion and metastases. MSLN expression and elevated serum soluble MSLN-related protein, generated after cleavage of MSLN by ADAM17, correlate with tumor burden and poor survival in TNBC [50]. The safety of transiently expressed (mRNA electroporation) second-generation MSLN CAR T cells was first evaluated in Phase I trials with no pleuritis, pericarditis or peritonitis reported (NCT01355965) [30]. Highly encouraging clinical activity including two metabolic complete responses have recently been reported in the context of mesothelioma in which stably engineered MSLN CAR T cells were administered by regional delivery (NCT02414269) [31]. MSLN-CAR T cells are currently being evaluated in Phase I/II studies (Table 2).

\section{Other antigens}

Tumor endothelial marker 8 (also known as anthrax toxin receptor 1, TEM8/ANTXR1), was initially described as a marker of tumor-associated endothelium in colorectal cancer. It has also been shown to be expressed in TNBC and TEM8/ANTXR1-specific CAR T cells control TNBC growth both in vitro and in vivo [35].

Folate receptor $\alpha(\mathrm{FR} \alpha)$ is highly expressed in nonmucinous epithelial tumors including breast cancer. Monoclonal antibodies have been used to redirect T cells or activating cytokines to FR $\alpha+$ tumors. FR $\alpha$-CAR T cells have also been reported to inhibit tumor cell growth in vitro and in vivo [36].

Other targets have shown tumor activity in preclinical models and are now being tested in Phase I trials such as CD70, EpCAM, CD44v6 and ROR1 (Table 2) [40,52,53]. 
(A)

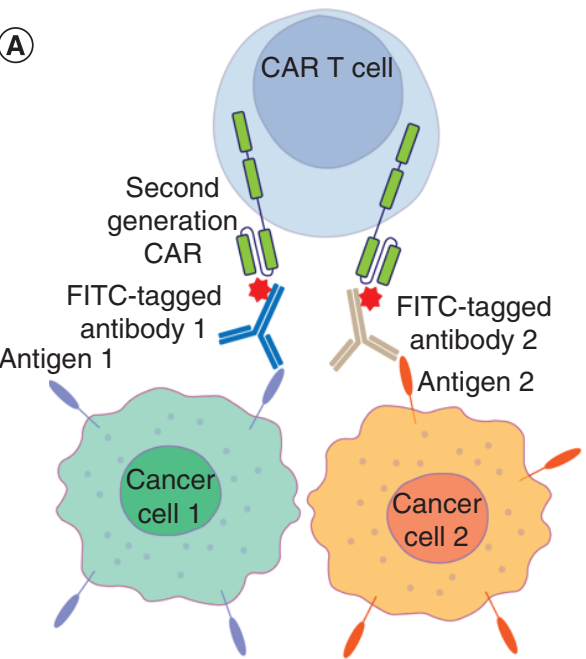

(B)

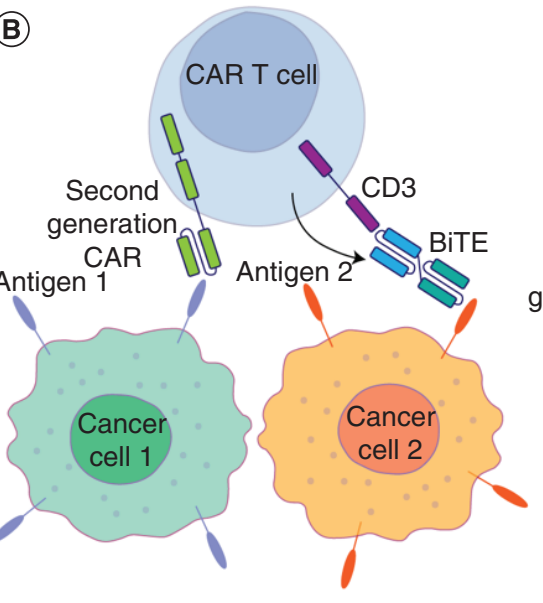

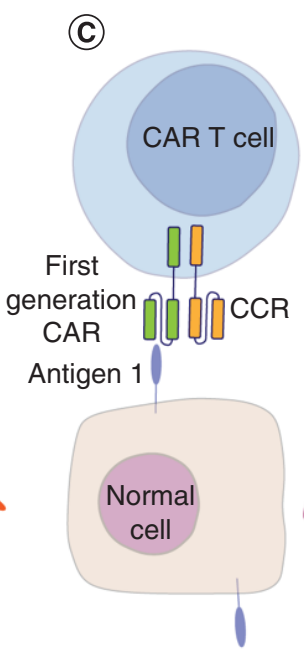

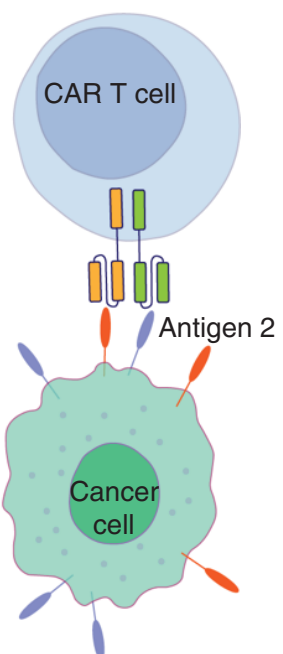

Figure 1. Engineering strategies to decrease tumor antigen escape and increase CAR T cell specificity. (A \& B) Reduced likelihood of tumor escape via antigen loss may conceptually be achieved by simultaneous recognition of two antigens by one universal (e.g., anti-FITC) CAR through the administration of two FITC-tagged antibodies or via the secretion of a bispecific reagent such as a BiTE by the CAR T cell. (C) Increased specificity is achieved by co-transducing a first generation CAR delivering signal 1 and a CCR delivering signal 2.

\section{Simultaneous targeting of multiple antigens}

Targeting of CAR T cells against a single antigen could favor immune escape in highly heterogeneous tumors. In order to prevent escape, strategies enabling the recognition of multiple antigens by a single CAR are being developed. Administration of biotinylated antibodies enable simultaneous or sequential recognition of multiple antigens by an avidin-based second generation CAR [54]. This approach has been tested on HER2 ${ }^{+}$and folate receptor positive cells using an anti-fluorescein isothiocyanate (FITC) CAR T cells in combination with intravenously administered FITC-HER2 antibodies or FITC-folate (Figure 1A) [37,38]. The secretion of bispecific T cell engagers (BiTE) by CAR T cells could also be used to prevent immune escape (Figure 1B) [25]. In a murine glioblastoma model, secretion of EGFR-CD3 BiTE by EGFRvIII-CAR T cells improves antitumor effect through the recruitment of bystander T cells. Importantly, local delivery of these cells could eliminate EGFRvIII-tumors. Switchable CARs have also been developed in which activity of the engineered $\mathrm{T}$ cell is controlled by an antibody-based switch molecule. Preclinical proof of concept for applicability in breast cancer models has been demonstrated [5].

Indirect antitumor effect may also be achieved through the targeting of nonmalignant cells such as cancerassociated stromal cells. Illustrating this, CAR T cells targeting the FAP have been shown to preferentially target FAP ${ }^{\text {high }}$ stromal cells and inhibit growth of several tumor cell types including breast carcinoma [39].

\section{Safety \& precision targeting}

Following CAR T-cell infusion, toxicity occurs mainly through two mechanisms. First, systemic release of high levels of cytokines may occur after T-cell activation. This can lead to cytokine release syndrome which results in hemodynamic instability, fever, hypoxia and seizures in severe cases, and requires treatment with tocilizumab (anti-IL-6 receptor monoclonal antibody) and/or corticosteroids. The second mechanism is on-target off-tumor toxicity involving target recognition on nonmalignant cells by the CAR.

Safety concerns were raised after the case report of a HER2-positive colon cancer patient treated with 4D5 third generation HER2 CAR T cells. The patient developed respiratory distress shortly after $10^{10} \mathrm{CAR} \mathrm{T}$ cells were infused and death followed within a few days [3]. Increased levels of serum cytokines were measured $4 \mathrm{~h}$ after infusion and accumulation of CAR T cells in the lungs and lymph nodes but not in lung metastases was documented postmortem. Toxicity was attributed to on target off tumor recognition of HER2 expressed by lung parenchymal or endothelial cells. The absence of CAR accumulation in the metastases despite systemic activation of the CAR T cells highlights the need to assess and optimize tumor infiltration as discussed later in this review. 
Subsequently, a second generation FRP5 HER2 CAR was tested in sarcoma patients at a lower dose (maximum $10^{8} / \mathrm{m}^{2}$ ) and in the absence of lymphodepleting chemotherapy or IL-2 support. No toxicity was reported and neither T-cell expansion nor IFN- $\gamma$ production were documented in peripheral blood although CAR T cells could be detected by qPCR for up to 18 months [4]. FRP5 and 4D5 bind different epitopes and FRP5 CAR T cells could be less prone to activation in response to ErbB2 expressed on nonmalignant cells. A direct comparison between these two CARs would be required to assess safety profiles accurately.

Transient expression of the CAR limits the risk of severe toxicity and offers a potential window for the attainment of antitumor efficacy. Tchou et al. studied the efficacy of mRNA electroporated cMET CAR T cells after intratumoral injection in breast cancer patients [29]. No toxicity above grade I was reported. Cutaneous metastases were resected after treatment and pathology revealed extensive necrosis and loss of cMET immunostaining suggesting that intratumoral injection of transiently CAR expressing $\mathrm{T}$ cells could be a safe and effective way to induce regression of accessible metastases. Alternatively, use of switchable CAR systems as described above provides an opportunity for titratable control of therapeutic activity.

Another strategy to increase specificity of CAR T-cell activation entails the use of a combination of chimeric receptors that deliver either signal 1 or signal 2 ('gated' CARs). Wilkie et al. demonstrated that simultaneous targeting of HER2 (signal 1) and MUC1 (signal 2) increased proliferation of dual-specific CAR T cells in the presence of target cells expressing both antigens (Figure 1C) [20].

Finally, in order to discriminate between cancer cells overexpressing TAAs and normal cells expressing low levels of these antigens, Liu et al. studied the activation of CAR T cells bearing affinity tuned CARs [6]. The authors show that reducing the affinity of the single chain antibodies for the HER2 and EGFR targets prevented CAR T-cell activation in response to weak but not high antigen expression, hence improving therapeutic index. This strategy might be further improved by using a combinatorial approach to deliver signal 1 and 2 .

\section{Addressing the immunosuppressive microenvironment}

In order to retain antitumor activity in the TME, CAR T cells need to overcome inhibitory signals delivered through immune checkpoint ligands or inhibitory cytokines [55]. PD-1 (expressed on T cells) and its ligand PD-L1 (predominantly expressed on tumor or stromal cells) constitute the most studied inhibitory axis in antitumor responses [56]. Upregulation of PD-1 ligands by tumor cells is one key mechanism of immune evasion as binding of PD-L1 to PD-1 plays a central role in T lymphocyte exhaustion. The administration of monoclonal antibodies binding the inhibitory receptors or their ligands has led to dramatic responses in melanoma and other solid tumors [56].

In a HER2 transgenic mouse model of breast carcinoma, antibody-mediated PD-1 blockade in combination with HER2 CAR T-cell treatment improved tumor control [7]. No increased toxicity was documented in normal $\mathrm{HER}^{+}{ }^{+}$tissues. In a prostate cancer mouse model, a helper dependent adenovirus encoding a PD-L1 blocking mini-body increased tumor control in combination with HER2 CAR T cells and an oncolytic adenovirus but not in combination with the oncolytic virus only (Figure 2A) [8].

Strategies avoiding the secretion of soluble PD-1 blocking molecules are also being developed. The concomitant transduction of a MSLN CAR with a dominant negative PD-1 receptor prevents functional impairment upon repeated antigen stimulation and this is associated with improved tumor control in a mouse model of mesothelioma [32,33]. A similar effect has been observed when a chimeric PD-1-CD28 receptor was co-transduced alongside an MSLN-CAR [34]. It has been shown in vitro that extracellular PD-1 fused with transmembrane and intracellular CD28 delivers costimulation which enhances the functionality of T cells (Figure 2B) [57].

IL-4 is another mediator involved in the tumor milieu immunosuppressive effect. Co-transduction of MUC1specific second generation CAR with a chimeric receptor comprising the extracellular IL-4R domain and the intracellular IL-7R domain improved tumor control in vitro and in vivo (Figure 2D) [19]. Another hybrid cytokine receptor, $4 \alpha \beta$, was developed to drive selective expansion of CAR T cells in the presence of IL- 4 . The ectodomain of IL- $4 \alpha$ receptor was fused with the $\beta$ common subunit which initiates intracellular signaling in response to IL-2 and IL-15 [18]. T cells transduced with this hybrid cytokine receptor alongside a MUC-1 targeting CAR expanded vigorously and retained the capacity to lyse cancer cells in vitro, even after multiple rounds of stimulation in the presence of IL-4.

Other approaches aiming at overcoming the immunosuppressive tumor environment rely on increased activation. The combination of 4-1BB agonist antibody and second generation HER2-CAR T lymphocytes improved tumor 

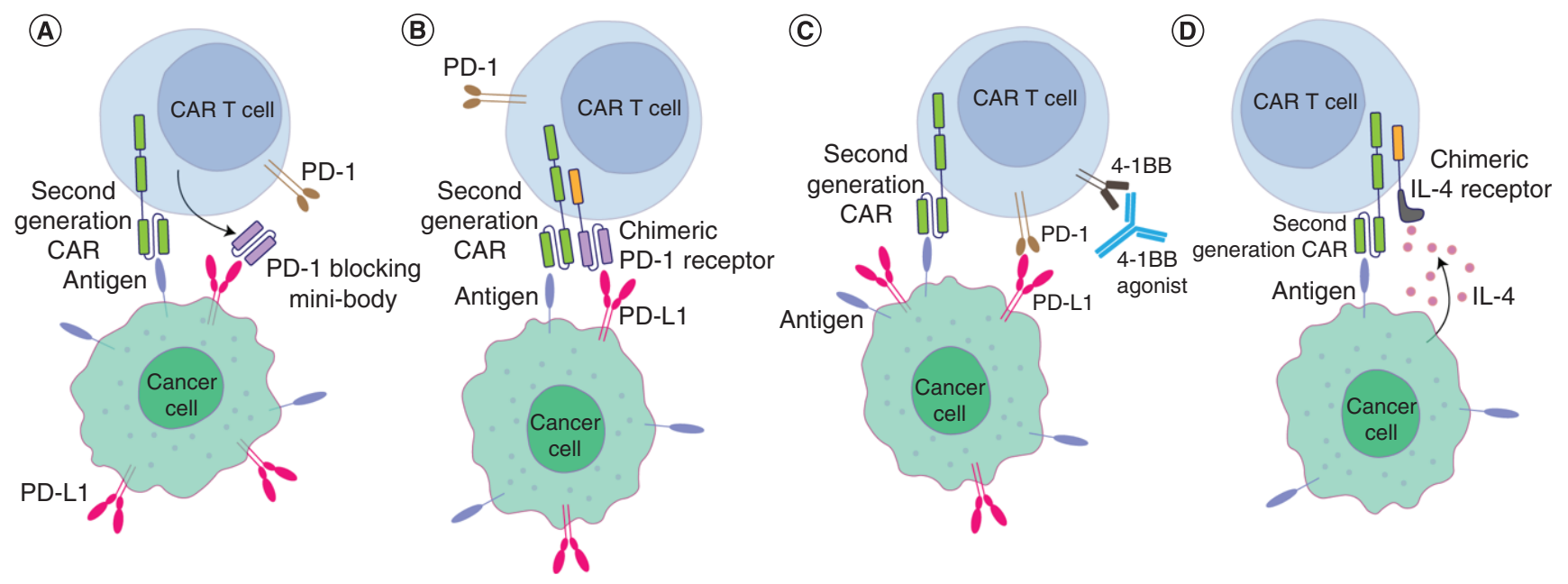

Figure 2. Strategies to overcome immunosuppression in the tumor microenvironment. (A \& B), Immunosuppression mediated by the PD-1/PD-L1 axis may be prevented by a secreted PD-L1 blocking mini-body or the expression of a chimeric receptor coupling PD-1 binding to an activating intracellular signal. (C) Increased co-stimulation using 4-1BB agonists circumvents T-cell exhaustion mediated by the PD-1/PD-L1 axis. (D) IL-4 delivers a proliferative signal when binding a chimeric IL-4 receptor comprising the IL-7 or IL-2/15R 3 intracellular domain.

control in vivo in a murine breast carcinoma model (Figure 2C) [9]. Increased CAR T-cell function was accompanied by reduced numbers of inhibitory leukocytes, including regulatory $\mathrm{T}$ cells and myeloid-derived suppressor cells.

The metabolic mediators of immune suppression in the TME and the metabolic requirements of CAR T cells for optimal in vivo efficacy have been the focus of attention in recent years. Depletion of nutrients (such as tryptophan, glutamine and glucose) and accumulation of immunosuppressive metabolites (such as kynurenine and nitric oxide) dampen intratumoral T-cell function [58]. It has also been shown that the metabolic optimization of CAR T-cell generation, through exposure to high concentration of arginine can improve in vivo antitumor activity in a mouse melanoma model [59]. Another mechanism of T-cell inhibition involves the adenosine $2 \mathrm{~A}$ receptor $\left(\mathrm{A}_{2 \mathrm{~A}} \mathrm{R}\right)$ which is expressed upon HER2 CAR T-cell activation and mediates the immunosuppressive effect of extracellular adenosine. Blockade of $A_{2 A} R$ has been shown to enhance tumor control $[10,60]$.

Finally, the transcriptional control of T-cell exhaustion is becoming better understood. Exhaustion due to intense CAR tonic signaling can be prevented by enforced expression of c-Jun, skewing the balance between AP-1 family homo and heterodimers toward the canonical c-Jun/c-Fos dimer which promotes IL-2 secretion [61]. Whether this approach could prevent exhaustion in breast cancer needs to be explored.

\section{Strategies to enhance CAR T-cell persistence}

Long-term persistence of CAR T cells is a key determinant of control of B cell malignancy and tends to correlate with the proportion of central memory $\left(\mathrm{T}_{\mathrm{CM}}\right)$ and stem cell memory $\left(\mathrm{T}_{\mathrm{SCM}}\right) \mathrm{T}$ cells in the CAR T-cell product [62]. Although these associations have been widely studied in the context of hematological malignancies, this needs further exploration is solid tumors such as breast cancer.

Co-stimulation is required for optimal CAR T cell function and persistence [63]. The best characterized costimulatory domains are CD28 and 4-1BB. Both increase antitumor activity but are associated with different CAR T-cell outcomes. Costimulation via 4-1BB favors the differentiation of CAR $\mathrm{T}$ cells into the central memory $\left(\mathrm{T}_{\mathrm{CM}}\right)$ subset whereas $\mathrm{CD} 28$ increases the proportion of effector memory $\left(\mathrm{T}_{\mathrm{EM}}\right) \mathrm{T}$ cells. Accompanying this, 4-1BB based CAR T cells demonstrate a greater propensity for oxidative metabolism in contrast to a predominantly glycolytic phenotype for CD28 based CAR T cells. The effect of CD27 costimulation on survival and antitumor activity has also been explored in a murine breast cancer model [64]. Nonetheless, the optimal form of costimulation required for breast cancer immunotherapy is still unknown.

Latent viral infections drive the long-term persistence of functional memory CD8 T cells due to intermittent low level antigenic stimulation [65]. Transduction of viral antigen-specific T cells has been explored as a strategy 
to increase persistence of CAR T cells. Treatment of mice bearing HER2 ${ }^{+}$sarcoma with HER2-CAR engineered T lymphocytes with specificity for Epstein Barr virus resulted in extended survival [11]. In a mouse model of breast cancer, treatment with bi-specific HER2-CAR and gp100-T cell receptor-engineered T lymphocytes in combination with gp100 encoding vaccinia virus achieved tumor eradication and prevented tumor engraftment upon re-challenge of cured mice [12]. A Phase I clinical trial assessed the safety of cytomegalovirus-specific cytotoxic T lymphocytes engineered to express a HER2-CAR in HER2 ${ }^{+}$glioblastoma patients (NCT00902044) [13]. CARpositive $\mathrm{T}$ cells persisted in peripheral blood for up to 12 months with no signs of systemic toxicity. Importantly, clinical benefit was observed in $50 \%$ of patients (partial response in one patient and stable disease in seven among 16 evaluated patients).

Concomitant administration of CAR T cells and vaccination has been shown to increase therapeutic activity against solid tumors. Claudin 6 (CLDN6) has been identified as a fetal antigen which can be aberrantly expressed in tumors. Using a liposomal RNA vaccine in combination with CLDN6-CAR T cells increased expansion of the transferred CAR T cells and eradication of established tumors by sub-therapeutic doses of CAR T cells in mouse models of lung and ovarian cancers [66]. A similar approach, based on amphiphile-CAR ligands decorating the surface of DCs, dramatically improved the anti-tumor effect of EGFRvIII-CAR T cells in a murine breast cancer model [24].

\section{Homing of CAR T cells}

Trafficking of CAR T cells to the site of disease is a limiting factor for CAR T cell anti-tumor activity. Injection of CAR T cells directly at the tumor site has been used to circumvent this hurdle, as described above in the study by Tchou et al. [29]. In a mouse model of breast cancer brain metastases, tumor eradication was achieved after intraventricular injection of second generation HER2-CAR T cells [14].

Combined approaches have also been used to increase intra-tumoral CAR T-cell trafficking. The administration of vasculature disrupting agents such as combretastatin A4 phosphate (CA4P) in combination with HER2-CAR $\mathrm{T}$ cells promoted infiltration in a murine model of SKOV3 [15]. In a murine breast carcinoma model of lung metastases, administration of IL-12 and doxorubicin upregulated the CXCL-9 and CXCL-10 chemokines, leading to increased $\mathrm{T}$ cell tumor infiltration and effector/regulatory T-cell ratio [67]. In order to avoid the risk of toxicity associated with systemic administration of cytokines, CAR T cells have been engineered to secrete factors promoting intratumoral trafficking. Co-expression of IL-7 and CCL-19 in CAR T cells significantly improved tumor clearance in a mastocytoma mouse model [68]. Contribution of recipient immune cells is suggested by the reduced tumor control observed when immunodepletion was performed prior to CAR T-cell treatment. In line with the previous observation, co-transduction of a constitutively active IL-7 receptor increased trafficking and tumor control in TNBC in vivo [41]. In a murine breast cancer model, expression of IL-33 in the TME also impaired tumor growth through enhancement of type 1 immune responses [69]. In this study, both NK and CD8 T cells were required to mediate IL-33 tumor control. Finally, IL-8 is secreted by several breast cancer cell lines such as the TNBC MDA-MB-468 and MDA-MB-231 cell lines. CAR T cells engineered to express CXCR1 or CXCR2, the IL-8 receptors, displayed enhanced intratumor migration and persistence which were associated with superior antitumor activity in glioma, ovarian and pancreatic cancer xenograft models [70,71]. Although the homing effect of IL-8 has not been assessed in breast cancer in vivo models, this might be a promising solution to increase trafficking to sites of disease.

\section{Future perspective}

A significant unmet therapeutic need remains for refractory and TNBC. Intense efforts are being made to overcome hurdles impairing CAR $\mathrm{T}$ cell safety and efficiency and refined engineering strategies have already improved CAR T-cell specificity, functionality, persistence and tumor infiltration in preclinical models. However, addressing the heterogeneity of breast cancers in patients remains a challenge $[44,45]$. The density and quality of immune infiltration in specific cancer subtypes are becoming better characterized. Also, the role of intratumoral dendritic and NK cells in the recruitment of adoptively transferred T lymphocytes has been identified in recent years [7274]. Developing clinically relevant immunocompetent animal models recapitulating these mechanisms will greatly improve preclinical development of adoptive cell therapy. Finally, a detailed understanding of tumor biology and the determinants of $\mathrm{T}$ cell trafficking into solid tumors in patients will be crucial to achieve tumor control. Based on this knowledge, combined approaches delivering CAR T cells while shaping the tumor immune infiltrate will be developed and unveil the full potential of CAR T cell therapy in solid tumors. 
Financial \& competing interests disclosure

Research in J Maher's laboratory is supported by Leucid Bio, Breast Cancer Now, British Lung Foundation, the Wellcome Trust, the J P Moulton Charitable Foundation, the King's Health Partners Research and Development Fund, the Experimental Cancer Medicine Centre at King's College London, the Cancer Research UK Centre at King's Health Partners and by the National Institute for Health Research (NIHR) Biomedical Research Centre based at Guy's and St Thomas' NHS Foundation Trust and King's College London. The views expressed are those of the authors and not necessarily those of the NHS, the NIHR or the Department of Health. J Maher is founder, chief scientific officer and shareholder in Leucid Bio. P Antoine has no conflicts of interest to report. The authors have no other relevant affiliations or financial involvement with any organization or entity with a financial interest in or financial conflict with the subject matter or materials discussed in the manuscript apart from those disclosed.

No writing assistance was utilized in the production of this manuscript.

\section{Open access}

This work is licensed under the Attribution-NonCommercial-NoDerivatives 4.0 Unported License. To view a copy of this license, visit http://creativecommons.org/licenses/by-nc-nd/4.0/

\section{References}

Papers of special note have been highlighted as: $\bullet$ of interest; $\bullet \bullet$ of considerable interest

1. Stancovski I, Schindler DG, Waks T, Yarden Y, Sela M, Eshhar Z. Targeting of T lymphocytes to Neu/HER2-expressing cells using chimeric single chain Fv receptors. J. Immunol. 151(11), 6577-6582 (1993).

\section{- Describes a HER2 CAR for the first time.}

2. Liu X, Zhang N, Shi H. Driving better and safer HER2-specific CARs for cancer therapy. Oncotarget 8(37), 62730-62741 (2017).

3. Morgan RA, Yang JC, Kitano M, Dudley ME, Laurencot CM, Rosenberg SA. Case report of a serious adverse event following the administration of $\mathrm{t}$ cells transduced with a chimeric antigen receptor recognizing ERBB2. Mol. Ther. 18(4), 843-851 (2010).

4. Ahmed N, Brawley VS, Hegde M et al. Human epidermal growth factor receptor 2 (HER2)-specific chimeric antigen receptor-modified T cells for the immunotherapy of HER2-positive sarcoma. J. Clin. Oncol. 33(15), 1688-1696 (2015).

5. Cao Y, Rodgers DT, Du J et al. Design of switchable chimeric antigen receptor T cells targeting breast cancer. Angew. Chemie - Int. Ed. 55(26), 7520-7524 (2016).

6. Liu X, Jiang S, Fang C et al. Affinity-tuned ErbB2 or EGFR chimeric antigen receptor T cells exhibit an increased therapeutic index against tumors in mice. Cancer Res. 75(17), 3596-3607 (2015).

7. John LB, Devaud C, Duong CPM et al. Anti-PD-1 antibody therapy potently enhances the eradication of established tumors by gene-modified T cells. Clin. Cancer Res. 19(20), 5636-5646 (2013).

8. Tanoue K, Shaw AR, Watanabe N et al. Armed oncolytic adenovirus-expressing PD-L1 mini-body enhances antitumor effects of Chimeric antigen receptor t cells in solid tumors. Cancer Res. 77(8), 2040-2051 (2017).

9. Mardiana S, John LB, Henderson MA et al. A multifunctional role for adjuvant anti-4-1BB therapy in augmenting antitumor response by chimeric antigen receptor T cells. Cancer Res. 77(6), 1296-1309 (2017).

10. Beavis PA, Henderson MA, Giuffrida $L$ et al. Targeting the adenosine $2 \mathrm{~A}$ receptor enhances chimeric antigen receptor $\mathrm{T}$ cell efficacy. $J$. Clin. Invest. 127(3), 929-941 (2017).

11. Nakazawa Y, Huye LE, Salsman VS et al. PiggyBac-mediated cancer immunotherapy using EBV-specific cytotoxic T-cells expressing HER2-specific chimeric antigen receptor. Mol. Ther. 19(12), 2133-2143 (2011).

12. Slaney CY, Von Scheidt B, Davenport AJ et al. Dual-specific chimeric antigen receptor T cells and an indirect vaccine eradicate a variety of large solid tumors in an immunocompetent, self-antigen setting. Clin. Cancer Res. 23(10), 2478-2490 (2017).

13. Ahmed N, Brawley V, Hegde $\mathrm{M}$ et al. HER2-specific chimeric antigen receptor-modified virus-specific $\mathrm{T}$ cells for progressive glioblastoma: a Phase II dose-escalation trial. JAMA Oncol. 3(8), 1094-1101 (2017).

14. Priceman SJ, Tilakawardane D, Jeang B et al. Regional delivery of chimeric antigen receptor-engineered $\mathrm{T}$ cells effectively targets HER2+ breast cancer metastasis to the brain. Clin. Cancer Res. 24(1), 95-105 (2018).

15. Deng C, Zhao J, Zhou S et al. The vascular disrupting agent CA4P improves the antitumor efficacy of CAR-T cells in preclinical models of solid human tumors. Mol. Ther. 28(1), 75-88 (2019).

16. Wilkie S, Picco G, Foster J et al. Retargeting of human T cells to tumor-associated MUC1: the evolution of a chimeric antigen receptor. J. Immunol. 180(7), 4901-4909 (2008).

- Describes a MUC1 CAR for the first time.

17. Posey AD, Schwab RD, Boesteanu AC et al. Engineered CAR T cells targeting the cancer-associated Tn-glycoform of the membrane mucin MUC1 control adenocarcinoma. Immunity 44(6), 1444-1454 (2016). 
18. Wilkie S, Burbridge SE, Chiapero-Stanke L et al. Selective expansion of chimeric antigen receptor-targeted T-cells with potent effector function using interleukin-4. J. Biol. Chem. 285(33), 25538-25544 (2010).

19. Bajgain $\mathrm{P}$, Tawinwung $\mathrm{S}, \mathrm{D}$ 'Elia $\mathrm{L}$ et al. CAR $\mathrm{T}$ cell therapy for breast cancer: harnessing the tumor milieu to drive $\mathrm{T}$ cell activation. $J$. Immunother. Cancer. 6(1), 1-13 (2018).

20. Wilkie S, Van Schalkwyk MCI, Hobbs $S$ et al. Dual targeting of ErbB2 and MUC1 in breast cancer using chimeric antigen receptors engineered to provide complementary signaling. J. Clin. Immunol. 32(5), 1059-1070 (2012).

21. Le Zuo B, Yan B, Zheng GX et al. Targeting and suppression of HER3-positive breast cancer by T lymphocytes expressing a heregulin chimeric antigen receptor. Cancer Immunol. Immunother. 67(3), 393-401 (2018).

22. Liu Y, Zhou Y, Huang K-H et al. EGFR-specific CAR-T cells trigger cell lysis in EGFR-positive TNBC. Aging (Albany. NY) 11(23), 11054-11072 (2019).

23. Davies DM, Foster J, van der Stegen SJC et al. Flexible targeting of ErbB dimers that drive tumorigenesis by using genetically engineered T cells. Mol. Med. 18(4), 565-576 (2012).

24. Ma L, Dichwalkar T, Chang JYH et al. Enhanced CAR-T cell activity against solid tumors by vaccine boosting through the chimeric receptor. Science 365(6449), 162-168 (2019).

-• Shows that CAR ligands decorating dendritic cells can be used to improve CAR T cell antitumor effect in vivo.

25. Choi BD, Yu X, Castano AP et al. CAR-T cells secreting BiTEs circumvent antigen escape without detectable toxicity. Nat. Biotechnol. 37(9), 1049-1058 (2019).

- Shows that CAR T cells can induce responses to multiple antigens through the secretion of bispecific $T$ cell engagers.

26. Han Y, Xie W, Song DG, Powell DJ. Control of triple-negative breast cancer using ex vivo self-enriched, costimulated NKG2D CAR T cells. J. Hematol. Oncol. 11(1), 1-13 (2018).

27. Frigault MJ, Lee J, Basil MC et al. Identification of chimeric antigen receptors that mediate constitutive or inducible proliferation of $\mathrm{T}$ cells. Cancer Immunol. Res. 3(4), 356-367 (2015).

28. Thayaparan T, Petrovic RM, Achkova DY et al. CAR T-cell immunotherapy of MET-expressing malignant mesothelioma. Oncoimmunology 6(12), 1-12 (2017).

29. Tchou J, Zhao Y, Levine BL et al. Safety and efficacy of intratumoral injections of chimeric antigen receptor (CAR) T cells in metastatic breast cancer. Cancer Immunol. Res. 5(12), 1152-1161 (2017).

30. Beatty GL, Haas AR, Maus MV et al. Mesothelin-specific chimeric antigen receptor mRNA-engineered T cells induce anti-tumor activity in solid malignancies. Cancer Immunol. Res. 2(2), 112-120 (2014).

31. Adusumilli PS, Zauderer MG, Rusch VW et al. Abstract CT036: a Phase I clinical trial of malignant pleural disease treated with regionally delivered autologous mesothelin-targeted CAR T cells: Safety and efficacy. Cancer Res. 79(Suppl. 13), CT036 LP-CT036 (2019).

32. Chen N, Morello A, Tano Z, Adusumilli PS. CAR T-cell intrinsic PD-1 checkpoint blockade: a two-in-one approach for solid tumor immunotherapy. Oncoimmunology 6(2), 1-3 (2017).

33. Cherkassky L, Morello A, Villena-vargas J et al. Human CAR T cells with cell-intrinsic PD-1 checkpoint blockade resist tumor-mediated inhibition Find the latest version: Human CAR T cells with cell-intrinsic PD-1 checkpoint blockade resist tumor-mediated inhibition. J. Clin. Invest. 126(8), 3130-3144 (2016).

34. Liu X, Ranganathan R, Jiang S et al. A chimeric switch-receptor targeting PD1 augments the efficacy of second-generation CAR T cells in advanced solid tumors. Cancer Res. 76(6), 1578-1590 (2016).

35. Byrd TT, Fousek K, Pignata A et al. TEM8/ANTXR1-specific CAR T cells as a targeted therapy for triple-negative breast cancer. Cancer Res. 78(2), 489-500 (2018).

36. Song DG, Ye Q, Poussin M, Chacon JA, Figini M, Powell DJ. Effective adoptive immunotherapy of triple-negative breast cancer by folate receptor-alpha redirected CAR T cells is influenced by surface antigen expression level. J. Hematol. Oncol. 9(1), 1-12 (2016).

37. Tamada K, Geng D, Sakoda Y et al. Redirecting gene-modified $\mathrm{T}$ cells toward various cancer types using tagged antibodies. Clin. Cancer Res. 18(23), 6436-6445 (2012).

38. Lee YG, Marks I, Srinivasarao M et al. Use of a single CAR T cell and several bispecific adapters facilitates eradication of multiple antigenically different solid tumors. Cancer Res. 79(2), 387-396 (2019).

39. Wang LCS, Lo A, Scholler J et al. Targeting fibroblast activation protein in tumor stroma with chimeric antigen receptor $\mathrm{T}$ cells can inhibit tumor growth and augment host immunity without severe toxicity. Cancer Immunol. Res. 2(2), 154-166 (2014).

40. Wallstabe L, Göttlich C, Nelke LC et al. ROR1-CAR T cells are effective against lung and breast cancer in advanced microphysiologic 3D tumor models. JCI Insight 4(18), e126345 (2019).

41. Zhao Z, Li Y, Liu W, Li X. Engineered IL-7 receptor enhances the therapeutic effect of AXL-CAR-T cells on triple-negative breast cancer. Biomed. Res. Int. 2020, 1-13 (2020).

42. Foley J, Nickerson NK, Nam S et al. EGFR signaling in breast cancer: bad to the bone. Semin. Cell Dev. Biol. 21(9), 951-960 (2010).

43. Krishnamurti U, Silverman JF. HER2 in breast cancer: a review and update. Adv. Anat. Pathol. 21(2), 100-107 (2014). 
44. Godoy-Ortiz A, Sanchez-Muñoz A, Parrado MRC et al. Deciphering her2 breast cancer disease: biological and clinical implications. Front. Oncol. 9, 1124 (2019).

45. da Silva JL, Cardoso Nunes NC, Izetti P, de Mesquita GG, de Melo AC. Triple negative breast cancer: a thorough review of biomarkers. Crit. Rev. Oncol. Hematol. 145, 102855 (2020).

46. Del Vecchio CA, Jensen KC, Nitta RT, Shain AH, Giacomini CP, Wong AJ. Epidermal growth factor receptor variant III contributes to cancer stem cell phenotypes in invasive breast carcinoma. Cancer Res. 72(10), 2657-2671 (2012).

47. Zhang Y, Su H, Rahimi M, Tochihara R, Tang C. EGFRvIII-induced estrogen-independence, tamoxifen-resistance phenotype correlates with PgR expression and modulation of apoptotic molecules in breast cancer. Int. J. Cancer 125(9), 2021-2028 (2009).

48. Lazarova M, Steinle A. Impairment of NKG2D-mediated tumor immunity by TGF- $\beta$. Front. Immunol. 10, 2689 (2019).

49. Meng F, Wu L, Dong L et al. EGFL9 promotes breast cancer metastasis by inducing cMET activation and metabolic reprogramming. Nat. Commun. 10(1), 5033 (2019).

50. Tozbikian G, Brogi E, Kadota K et al. Mesothelin expression in triple negative breast carcinomas correlates significantly with basal-like phenotype, distant metastases and decreased survival. PLoS One 9(12), 1-16 (2014).

51. Pastan I, Pai LH, Batra JK, Pastan I, Willingham MC. Characterization of the antigen (CAK1) recognized by monoclonal antibody Kl present on ovarian cancers and normal mesothelium. Cancer Res. 52(1), 181-186 (1992).

52. Petrau C, Cornic M, Bertrand P et al. CD70: a potential target in breast cancer? J. Cancer 5(9), 761-764 (2014).

53. Liu L, Yin B, Yi Z et al. Breast cancer stem cells characterized by CD70 expression preferentially metastasize to the lungs. Breast Cancer 25(6), 706-716 (2018).

54. Urbanska $\mathrm{K}$, Lanitis E, Poussin $\mathrm{M}$ et al. A universal strategy for adoptive immunotherapy of cancer through use of a novel T-cell antigen receptor. Cancer Res. 72(7), 1844-1852 (2012).

55. Martinez M, Moon EK. CAR T cells for solid tumors: new strategies for finding, infiltrating, and surviving in the tumor microenvironment. Front. Immunol. 10, 1-21 (2019).

56. Zou W, Wolchok JD, Chen L. PD-L1 (B7-H1) and PD-1 pathway blockade for cancer therapy: mechanisms, response biomarkers, and combinations. Sci. Transl. Med. 8(328), 328rv4 (2016).

57. Prosser ME, Brown CE, Shami AF, Forman SJ, Jensen MC. Tumor PD-L1 co-stimulates primary human CD8+ cytotoxic T cells modified to express a PD1: CD28 chimeric receptor. Mol. Immunol. 51(3-4), 263-272 (2012).

58. Xu X, Rashida Gnanaprakasam JN, Sherman J, Wang R. A metabolism toolbox for CAR T therapy. Front. Oncol. 9, 322 (2019).

59. Geiger R, Rieckmann JC, Wolf $\mathrm{T}$ et al. L-arginine modulates $\mathrm{T}$ cell metabolism and enhances survival and anti-tumor activity. Cell 167(3), 829-842.e13 (2016).

60. Hammami A, Allard D, Allard B, Stagg J. Targeting the adenosine pathway for cancer immunotherapy. Semin. Immunol. 42, 101304 (2019).

61. Lynn RC, Weber EW, Sotillo E et al. c-Jun overexpression in CAR T cells induces exhaustion resistance. Nature 576(7786), 293-300 (2019).

-. Demonstrates the possibility to harness transcriptional regulation to prevent exhaustion of CAR T cells.

62. Morgan MA, Schambach A. Engineering CAR-T cells for improved function against solid tumors. Front. Immunol. 9 , 2493 (2018).

63. Rafiq S, Hackett CS, Brentjens RJ. Engineering strategies to overcome the current roadblocks in CAR T cell therapy. Nat. Rev. Clin Oncol. 17(3), 147-167 (2020).

64. Song DG, Ye Q, Poussin M, Harms GM, Figini M, Powell DJ. CD27 costimulation augments the survival and antitumor activity of redirected human T cells in vivo. Blood 119(3), 696-706 (2012).

65. Marchi E, Lee LN, Klenerman P. Inflation vs. exhaustion of antiviral CD8+ T-cell populations in persistent infections: two sides of the same coin? Front. Immunol. 10, 197 (2019).

66. Reinhard K, Rengstl B, Oehm P et al. An RNA vaccine drives expansion and efficacy of claudin-CAR-T cells against solid tumors. Science 367(6476), 446-453 (2020).

-. Shows that RNA vaccines can lead to CAR T-cell expansion an improved tumor control.

67. Hu J, Sun C, Bernatchez C et al. T-cell homing therapy for reducing regulatory T cells and preserving effector T-cell function in large solid tumors. Clin. Cancer Res. 24(12), 2920-2934 (2018).

68. Adachi K, Kano Y, Nagai T, Okuyama N, Sakoda Y, Tamada K. IL-7 and CCL19 expression in CAR-T cells improves immune cell infiltration and CAR-T cell survival in the tumor. Nat. Biotechnol. 36(4), 346-351 (2018).

69. Gao X, Wang X, Yang Q et al. Tumoral expression of IL-33 inhibits tumor growth and modifies the tumor microenvironment through CD8+ T and NK cells. J. Immunol. 194(1), 438-445 (2015).

70. Whilding LM, Halim L, Draper B et al. CAR T-cells targeting the integrin $\alpha \mathrm{v} \beta 6$ and co-expressing the chemokine receptor CXCR2 demonstrate enhanced homing and E ffi cacy against Several Solid Malignancies. Cancers (Basel) 11(674), 1-17 (2019). 
71. Jin L, Tao H, Karachi A et al. CXCR1- or CXCR2-modified CAR T cells co-opt IL-8 for maximal antitumor efficacy in solid tumors. Nat. Commun. 10(1), 1-13 (2019).

72. Bareche $\mathrm{Y}$, Buisseret L, Gruosso $\mathrm{T}$ et al. Unraveling triple-negative breast cancer tumor microenvironment heterogeneity: towards an optimized treatment approach. J. Natl Cancer Inst. 112(7), 708-719 (2020).

73. Barry KC, Hsu J, Broz ML et al. A natural killer-dendritic cell axis defines checkpoint therapy-responsive tumor microenvironments. Nat. Med. 24(8), 1178-1191 (2018).

-. Shows that cellular networks are involved in lymphocyte trafficking to solid tumors.

74. Spranger S, Dai D, Horton B, Gajewski TF. Tumor-residing Batf3 dendritic cells are required for effector T cell trafficking and adoptive T cell therapy. Cancer Cell 31(5), 711-723 (2017). 